\title{
Meta-model for Collaboration Modeling in Legal Sector
}

\author{
S. Vasanthapriyan ${ }^{1}$, R.P.T. Bhagya ${ }^{1}$, P. Jayaweera ${ }^{2}$ \\ ${ }^{1}$ Department of Computing and Information Systems, Sabaragamuwa University of Sri Lanka, \\ Sri Lanka \\ ${ }^{2}$ Department of Computer Science, University of Sri Jayewardenepura, Sri Lanka
}

\begin{abstract}
Cost effective and best practice legal services are highly relying on the coordination of collaborative workflow activities as well as of several resources needed to perform these activities and their flow of information exchanges among many different participants. In the context of ever increasing numbers of legal cases and involved stakeholders in multi-party collaborations, we have discovered the appropriateness of the adaptation of workflow management systems in legal sector to address the resulting complexities and performance issues in legal service collaborations. In this work, a meta-model for legal service collaboration modeling which includes the main semantics of modeling elements, has been introduced as the basis for defining the choreography for sector collaboration with the objective of facilitating legal collaboration modeling in such a way as to provide a useful input for the creation of legal workflow specifications for setting up legal workflow management systems. The meta-model was developed based on Business Transaction View meta-model in UN/CEFACT's recommendations for business collaborations. The proposed modeling framework could facilitate and guide the complex legal collaboration modeling processes with promising results in workflow coordination.
\end{abstract}

KEYWORDS: Meta-model, Legal Collaborations, Legal Activity Coordination 


\section{INTRODUCTION}

The legal sector is comprised of a diverse array of institutions and participants who contribute to legal cases and share case related information that are fundamental to obtain cost effective and best practice legal services. In this respect, coordination of various legal workflow activities, as well as of several resources needed to perform these activities and their flow of legal information exchanges, plays a central role to foster excellence legal services and to facilitate whole of sector collaboration. However, frequently created new legal cases and increased numbers of roles offering different legal services, have resulted complexities and performance issues in legal service collaborations.

A prominent application area where such complex multi-party collaborations are formalized is workflow management systems (WFMS) (Hollingsworth, 1994). In general, a WFMS supports design, execution and monitoring of business workflows that typically involve multiple activities and collaborating parties in a distributed environment. By arranging the workflow activities, WFMS promise to increase the efficiency of business processes and consequently, to raise the business effectiveness. It therefore is clear that, the adaptation of WFMS in legal sector could lead to overcome the complexity issues in multi-party collaborations, while enabling more efficient functionalities and improving the quality of legal services. Ordinarily, the highest level development architecture of a WFMS could be characterized into build-time and runtime functions. The build-time functions are concerned with defining and modeling workflow activities; while the run-time functions are concerned with managing workflow executions. Accordingly, users of a WFMS interact with workflow modeling techniques and methodologies to generate a workflow specification, which is then stored in a workflow repository and made available to a run-time service called workflow enactment service for execution.

Currently, there are several metamodels for business and workflow process modeling with the focus of supporting business collaboration. For instance, in electronic business designing, UN/CEFACT's (United Nation's Center for Trade Facilitation and Electronic Business) recommendations could be considered as a globally accepted standard. However during our literature survey, it was evident that there is no any work similar to UN/CEFACT's recommendations to be adopted in legal sector that could assist in modeling legal collaboration workflows. In this paper, we attempt to develop a meta-model based on UN/CEFACT recommendation that could be used as the basis for defining the choreography for legal sector collaborations with the objective of facilitating legal collaboration modeling in such a way as to provide a useful input for the creation of legal workflow specifications for setting up legal WFMS.

The paper is structured as follows. In section 2, we discuss the related works and describe the relevant concepts. In section 3, we have noted the adopted research methodologies. The proposed meta-model is presented and discussed in section 4. In section 5, we conclude the paper 


\subsection{Related work and background}

In this section, we have briefed the related work and the foundation of the proposed meta-model for legal collaboration modeling.

\section{Related Work}

- NCSC (National Center of State Courts) Some work could be found on NCSC (2014) in relation to case flow and workflow management. However these contributions are not directly related and addressed the legal service collaboration modeling specifically as such.

- NACM (National Association for Court Management) - Another collection of works on case flow management could be found at NACM (2014). However these technical standards are at very low technical level and not addressed on legal service collaboration modeling as of interest in our work.

\section{Modeling Language}

Constructing a workflow specification is not an easy task. Observations have shown that the hardest task in the development of a workflow specification is how the model can be developed with the concepts and notations that are truthful to the language of the models. Modeling Language (ML) is a notion that specifies all the elements with which any domain model can be described (Tolvanen, 1998). The use of a ML enables domain users to abstract and share knowledge, and makes it easier to model the workflow processes. More importantly, to describe the structure, behavior, and properties of models, a special model that is capable of describing the language of these models is required. A meta-model is a model that has the ability to create the ML of many domain models (Tolvanen, 1998). It could generalize most of the concepts used in domain models by unifying the views and structuring the language of the domain. Hence, our work focused on the development of a meta-model for legal service collaboration modeling which includes main semantics of modeling elements with the objective of facilitating the legal collaboration modeling to generate legal workflow specifications.

\section{UN/CEFACT Modeling Methodology}

For electronic business collaboration modeling, there is a huge collection of approaches. However, among them, UN/CEFACT Modeling Methodology (UMM) (UMM, 2014) is well known and adopted in many different industries. Mainly, UMM delivers a metamodel that facilitates the specification of reusable, reproducible process models that are technology and protocol insensitive and advices well defined workflows for business collaboration designs. As, the UMM metamodel consists of four views in order to describe the business collaboration models, our work focused on the Business Transaction View (BTV) (chapter 8 UMM, 2014), considering that, it defines the orchestration of the business collaboration and structures the business information exchanged.

\section{MATERIALS AND METHODS}

In literature, there are several paradigms that one could found information systems related research and development work. However, two popular complementary information systems related research methodologies are behavioral science and design science (Dawson, 2002). In simple terms, behavioral science focuses on 
build and verifies new theories to describe some organizational or human phenomena. On the other hand, design science focuses on applying knowledge and inventing new artifacts for human purposes. Further, there are several possible categorizations on popular research methodologies. Commonly available categorization scheme for research work in sciences and humanities is called Quantitative Research vs. Qualitative Research (Dawson, 2002). Quantitative research deals with quantities mainly based on statistical approaches to study quantitative properties. For qualitative researching, grounded theory and action research are two widely known approaches. In action research, solutions are proposed based on collected data and then solutions are evaluated to study the consequences. However, the approach that we took for the development process of our solution within this work could be considered as hybrid of design science and action research.

\section{Collaboration Modeling Meta-model}

To define the choreography of legal sector collaborations between multiple collaborating parties, a conceptual meta-model is proposed in this section by depicting compliance concepts and relations between them.

\section{Legal Domain View}

During our investigation in legal sector, we have noticed several significant differences between generic business and legal service collaborations that demand for a need on an extension to the original UN/CEFACT's proposal.

Accordingly, at first, we have defined the Legal Collaboration Map (LCM) based on
UN/CEFACT's Business Operations Map (BOM) framework (chapter 8 UMM, 2014) to discover the legal area sub-process interrelationships. Figure 1 illustrates the identified interrelationships between the LCM modeling elements. There, a legal process area could be considered as a category of legal processes and legal transactions, and a legal process could be viewed as a set of legal tasks performed by individual partners together with legal interface tasks performed collaboratively among two or more parties

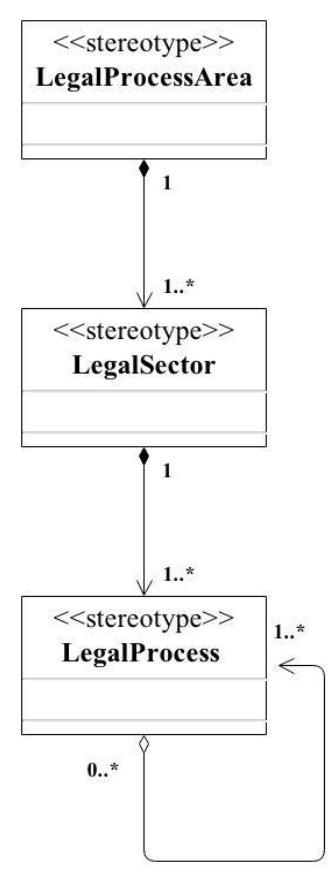

Figure 1. Interrelationships between LCM Modeling Elements

\section{Legal Transaction View}

In turn, we have developed a set of semantics in compliance with but by extending original model semantics of BTV that has been proposed in UN/CEFACT's recommendations. Figure 2 illustrates the proposed model elements that could be used to express the structure and behavior of objects for building 
the collaboration models in legal sector as well as Figure 3 illustrates their interrelationships.

In the following sub sections, we have briefed the proposed model elements.

\section{Legal Transaction}

There is an original UMM model element called Business Transaction that is used to represent a set of business information and business signal exchanges between two business partners which occurs in an agreed format, sequence and time period. In connection with this, a model element called Legal Transaction is proposed as an abstract class to represent a set of legal information and signal exchanges between two legal service partners in the legal sector.

\section{Legal Transaction Activity}

This specialized model element is very much analogous to the original UMM model element called, Business Transaction Activity. The requirement for adaptation of this element is to represent a legal service collaboration protocol activity that executes a specified legal transaction. This is also an abstract class that is not a stereotype.

\section{Legal Collaboration Protocol}

A legal collaboration protocol choreographs one or more legal transaction activities as in the original Business Collaboration Protocol model element in UMM recommendations. In this context, the proposed Legal Collaboration Protocol model element could be considered as an abstract class rather than a stereotype.

\section{Legal Service Partner}

This model element is very much identical to original Business Partner model element in UMM recommendations. The requirement for adaptation of this model element is to represent the partners that participate in legal service collaborations who are enumerated for each legal collaboration protocol. Such partners could provide the initiating and responding roles in the protocol. Similar to the above model elements, even the Legal Service Partner element is also an abstract class.

\section{Legal Action}

This model element is very much identical to original Business Action model element in UMM recommendations. The requirement for adaptation of this model element is to represent the legal actions executed by an authorized role. This is an abstract class that is not a stereotype. The state of a legal transaction could be defined by such reciprocal legal actions. However, during our investigation in legal sector, we get explored the need for differentiation of Legal Action model element into two as Responding Legal Activity and Requesting Legal Activity.

\section{Responding Legal Activity}

This specialized model element is very much analogous to the original UMM model element called, Responding Business Activity. The requirement for adaptation of this specialized element is to represent the legal activities that are performed by a partner role responding to another partner role's request for legal service. 


\section{Requesting Legal Activity}

Requesting Legal Activity model element is very much identical to the original UMM model element, Requesting Business Activity. The usage of this model element is to represent the legal activities that are performed by a partner role, requesting a legal service from another partner role. However, during our investigation in legal sector, we get explored the need for stating many different legal transaction activity elements which are specialized elements derived from the Requesting Legal Activity element as in UMM recommendations.

\section{Multilateral Offer Acceptance Activity}

This specialized model element could be considered as an extension to UMM Commercial Transaction Activity model element. The requirement for adaptation of this specialized element is to represent the establishment of a contract for governing collaboration with authorized roles.

\section{Request Response Activity}

There is a Request Response Activity model element in original UMM recommendations that use to represent the business activities of requesting data that need a complex calculation by the responding partner role. However, according to the sector requirement we were explored during our analysis, we get explored the need for differentiation of Request Response Activity model element into four sub categories that might represent different weightages in modeling legal proceedings. In general, the requirement for adaptation of these specialized model elements is representing the legal activities of requesting for information that the responding partner role not readily has or available but needs complex elicitation process to be executed in responding partner's end to provide the requested information.

\section{Information Querying Activity}

Information Querying Activity model element is very much identical to original Query Response Activity element that has been proposed in UN/CEFACT recommendations. The requirement for adaptation of this element is to represent the legal activities of querying the content that the responding partner role already has.

\section{Request Case Hearing Status Confirmation Activity}

There is a Request Confirm Activity model element in original UMM recommendation that uses to represent the activities of demanding the status of an agreement. The Request Case Hearing Status Confirmation Activity is much identical to this original model element. The requirement for adaptation of this specialized model element is to represent the legal activities of requesting confirmation about the status with respect to legal case proceedings.

\section{Request Made at Court Activity}

Request Made at Court Activity is a very special model element that is not common in generic business collaboration meta-model. During our investigation in legal sector, we get explored the need for differentiation of representing the requests made at a court by either party to a case into two. One is named as Request Order Activity, through which the requests to the court to make an order could be represented. The other is Request Grant Activity, through which the activities of requesting to the court to concede for conducting a specific legal proceeding could be modeled. 


\section{Order Completion Activity}

Order Completion Activity model element is also very special element that is not complying with the original model elements that has been proposed in UN/CEFACT recommendations. The requirement for adaptation of this specialized element is to represent the legal activities for ordering a specific legal proceeding with respect to ongoing cases.

\section{Case Hearing Information Notification} Activity There is an original UMM model element called, Notification Activity that could be used to represent the legal activities of notifying recipient with non-repudiation requirement. However, according to the sector requirements, we get explored the need for differentiation of Notification Activity into two. One model element is named as Direct Notification Activity, through which the legal activity that the intended recipient is directly informed with some notice is represented. In the meantime, we get noticed the need for a specialization of Direct Notification Activity model element, called Judgment Notification Activity. The other element is Indirect Notification Activity, which use to represent the legal activities for notifying the immediate recipient with intension of communicating to intended party of the notice.

\section{Authorized Role}

There is an original UMM model element called, Authorized Role that could be used to represent the partner roles that perform a functional role. Either an employee role or an organizational role can perform a functional role. Authorized Role model element is an Case Hearing Document which could be used to represent any document that is used at the case abstract class that is not a stereotype. However, during our investigation in legal sector, we get explored the need for differentiation of the representation of legal domain partner roles into three, as specialized elements derived from the Authorized Role model element. These specialized model elements are named as Court Room Personnel Role which could be used to represent the authorized roles involved in the court system, Plaintiff Party Role which could be used to represent the authorized roles engaged with the plaintiff party to a legal case and Defendant Party Role which could be used to represent the authorized roles involved in the defendant party to a legal case.

\section{Legal Document}

During our investigation, we have noticed that the partner roles always should exchange legal information in structured manner while performing many different legal activities. In this regard, Legal Document model element could be considered as an extension to original UMM Business Document model element. We have further specialized Legal Document modeling element into three sub-types according to the phases in a lawsuit that legal information is required. Therefore, the first subtype of Legal Document element is named as Case Filling Document which could be used to represent any document that is used to file a case in the court. The second sub-type of Legal Document model element is named as hearing processes. The third sub-type is named as Judge mental Document which could be used to represent any document that is used at the judgment issuing processes. 


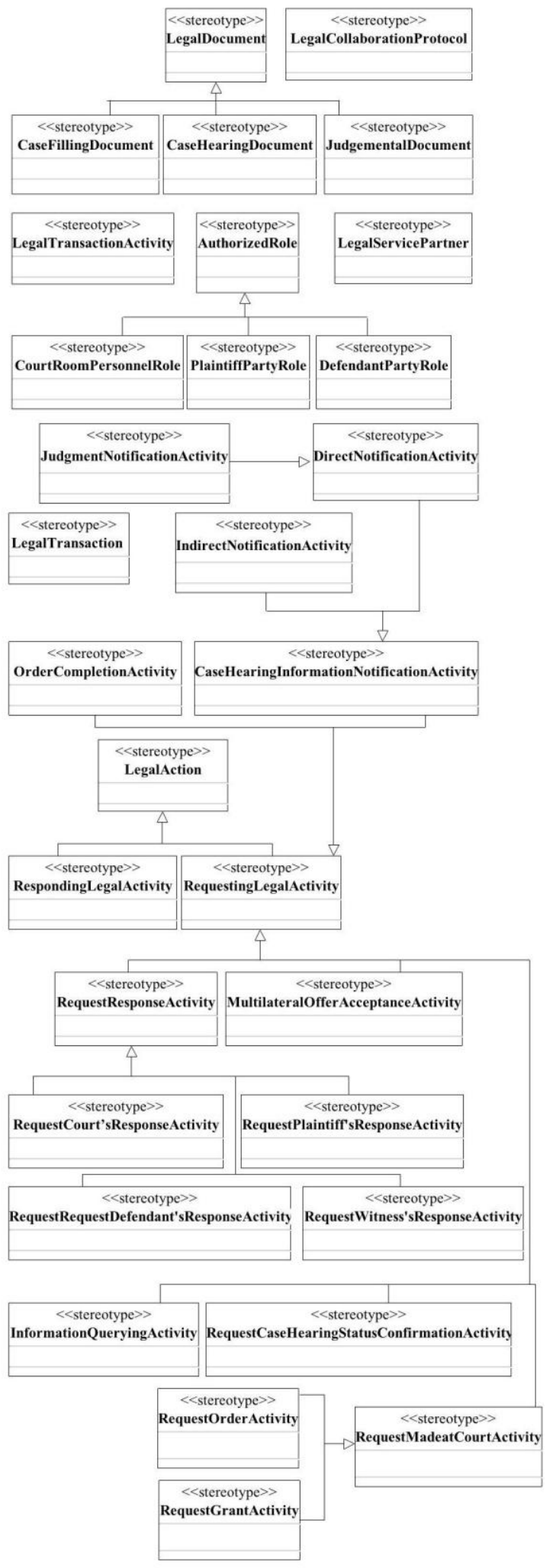




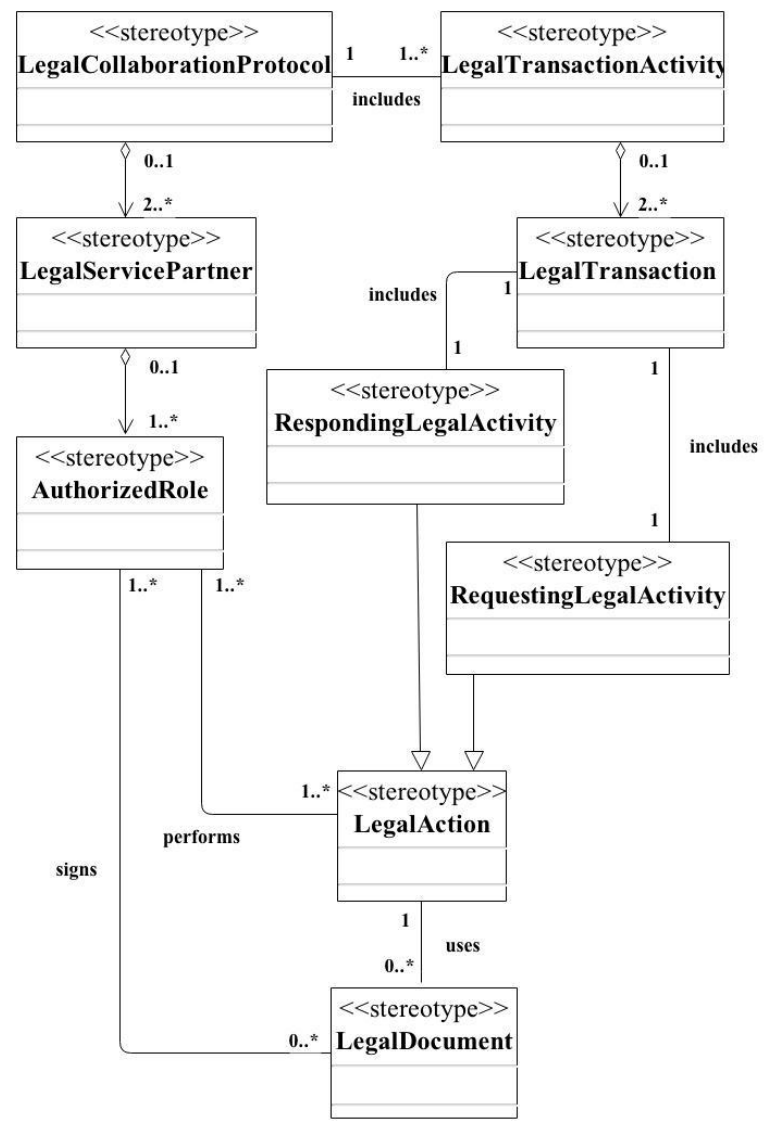

Figure 3. Interrelationships between Proposed Model Elements

\section{CONCLUSION}

In this paper, we have proposed a sound metamodel for legal service collaboration modeling with the objective of facilitating a modeling methodology to initiate the specification of legal workflows; as the foundational requirement for setting up a WFMS in legal sector. For the development of the meta-model, we have based the meta-model of BTV on globally accepted standard, UN/CEFACT's recommendations. With utilization of the proposed meta-model in designing complex multi-party legal collaborations, much of burdens connected with specification of legal work-flows could readily be overcome, since it clearly defines the choreography for sector collaboration as well as the structure of legal information exchanges.

Finally, we would like to brief some of the possible future directions of the work we reported here. Among them, with utmost priority is illustrating applications of the metamodel in legal collaboration modeling process following with complete empirical evaluations. 


\section{REFERENCES}

DAWSON, C. (2002). A Practical Guide to Research Methods: A user-friendly manual for mastering research techniques and projects. United Kingdom: How to Books.

HOLLINGSWORTH, D. (1994). Workflow Management Coalition. [Online] Available from:

http://www.aiai.ed.ac.uk/project/wfmc/.

[Accessed: 26th April 2014].

NACM. (2014). National Association for Court Management. [Online] Available from: https://nacmnet.org/. [Accessed: 26th April 2014].

NCSC. (2014). National Center of State Courts. [Online] Available from: http://www.ncsc.org/. [Accessed: 26th April 2014].

TOLVANEN, J. (1998). Incremental Method Engineering with Modeling Tools. [Online] Available from: http://users.jyu.fi/ jpt/Tolvanen_dissertation. pdf. [Accessed: 26th April 2014].

UMM. (2014) UMM Revision 10 (2001). [Online] Available from: http://www.unece.org/cefact/umm/umm_ind ex.html. [Accessed: 26th April 2014]. 